\title{
Induction chemotherapy in patients with resectable laryngeal cancer: A meta-analysis
}

\author{
PEI GAO ${ }^{1}$, LIANG GONG ${ }^{2}$ and XUEFENG WANG ${ }^{2}$ \\ ${ }^{1}$ Department of Otolaryngology, Jinzhou Medical University; ${ }^{2}$ Department of Otolaryngology, \\ The First Affiliated Hospital of Jinzhou Medical University, Jinzhou, Liaoning 121000, P.R. China
}

Received December 20, 2017; Accepted June 6, 2018

DOI: $10.3892 /$ mco.2018.1645

\begin{abstract}
Head and neck squamous cell carcinoma (SCC) ranks 6th among the most frequently diagnosed carcinomas globally. Laryngeal carcinoma is quite common, and $95 \%$ of the cases are SCCs. Since the introduction of larynx-preserving surgery, induction chemotherapy (IC) has played a substantial role. The aim of IC is to shrink or downstage primary laryngeal carcinomas, increasing the chances of complete surgical removal, particularly in cases with advanced but potentially resectable lesions. The aim of the present study was to investigate the value of IC in patients with resectable laryngeal cancer. A meta-analysis was performed of randomized controlled trials (1985-2017) investigating the effect of IC on survival, disease control, larynx-preserving surgery and disease-free survival. Engauge-Digitizer software was used to construct Kaplan-Meier curves and RevMan software was used for the analysis of the data. A total of 12 trials (4,320 patients) were included. There was no significant difference in local recurrence or locoregional control between patients receiving and those not receiving IC ( $\mathrm{P}>0.05)$. However, the experimental group (IC) exhibited a lower propensity for distant metastasis by $11.7 \%$ (95\% confidence interval: $10.3-13.3 \%, \mathrm{P}=0.02)$ compared with the control group (no IC). Among patients with laryngeal cancer, larynx preservation was possible in those who responded well to IC, without a significant decrease in survival compared with radical surgery $(\mathrm{P}<0.05)$. Taking into
\end{abstract}

Correspondence to: Professor Xuefeng Wang, Department of Otolaryngology, The First Affiliated Hospital of Jinzhou Medical University, No. 2, The Fifth Section of Renmin Street, Guta, Jinzhou, Liaoning 121000, P.R. China

E-mail: 765873648@qq.com

Abbreviations: HNSCC, head and neck squamous cell carcinoma; IC, induction chemotherapy; LC, laryngeal carcinoma; RCTs, randomized controlled trials; HR, hazard ratio; 95\% CI, 95\% confidence interval; LP, laryngeal preservation; DCs, dendritic cells; PF, cisplatin with 5-fluorouracil; EGFR, epidermal growth factor receptor; DFS, disease-free survival; GWAS, genome-wide association study

Key words: resectable laryngeal carcinoma, induction chemotherapy, randomized controlled trial, surgery, radiotherapy consideration these findings, IC confers an advantage in terms of lowering the risk of distant metastasis in patients with resectable laryngeal carcinoma, and enables laryngeal preservation in responders. Moreover, IC increases the overall survival rate in patients with locally advanced but resectable LC.

\section{Introduction}

Laryngeal carcinoma (LC) is a common tumor of the head and neck. LC patients at early clinical stages (I and II) are traditionally treated with surgery or radiotherapy, whereas patients with advanced-stage disease (III and IV) may require comprehensive sequential treatment combining surgery, radiotherapy and chemotherapy (1). However, despite aggressive treatment, no major improvement has been achieved in terms of prognosis, with a 5-year survival rate of 50-60\%, which is even lower in patients at advanced clinical stages (2).

Induction chemotherapy (IC) is considered as a reliable approach to controlling locally advanced LC, increasing the rate of laryngeal preservation and decreasing the risk of local spread or distant metastasis, thereby increasing the therapeutic efficacy. However, locoregional control must be performed in accordance with the tumor borders following IC, which should be marked at the start of the therapy (3), regardless of the response to IC. The clinical value of IC remains a matter of debate, particularly in cases with resectable advanced LC. Randomized controlled trials (RCTs) have reported conflicting results, whereas earlier systematic reviews have not demonstrated an obvious benefit of IC in terms of overall survival (OS) (4-7). There is currently no definitive evidence favoring IC with locoregional control over locoregional control alone for locally advanced and resectable LC. Accordingly, a meta-analysis of OS rate, local control, metastases and laryngeal preservation was conducted.

\section{Materials and methods}

Inclusion criteria. RCTs were considered eligible when they included formerly untreated patients with resectable non-metastatic LC, performing a comparison between IC followed by locoregional treatment (laryngectomy, or radiotherapy, or concomitant radiotherapy and chemotherapy, or laryngectomy combined with radiotherapy or chemoradiotherapy) and locoregional treatment alone. RCTs on laryngeal preservation were also considered as eligible if they performed 
a comparison between radical surgery together with radiotherapy vs. IC followed by radiotherapy or chemoradiotherapy in responders, or radical surgery with radiation therapy or chemoradiotherapy in non-responders. The included studies were articles published in English and they included patients treated between January 1, 1965 and December 31, 2017.

Exclusion criteria. The exclusion criteria were the following: i) Abstracts, letters, or meeting proceedings; ii) incomplete studies, studies with data duplication or not reporting outcomes of interest; iii) a case number of $<40$.

Search strategy. A systematic search was performed through Medline, EMBASE and The Cochrane Library for studies published up to June 1985 using a combination of the following search terms: Neoplasms, laryngeal/laryngeal neoplasm/neoplasm/laryngeal/larynx neoplasms/larynx neoplasm/neoplasm, larynx/neoplasms, larynx/cancer of larynx/ larynx cancers/laryngeal cancer/cancer, laryngeal/cancers, laryngeal/laryngeal cancers/larynx cancer/cancer, larynx/cancers, larynx/cancer of the larynx, chemotherapies, induction/chemotherapy, induction/induction chemotherapies, laryngectomies/ laryngectomy/radiation oncology/oncology, radiation/therapeutic radiology/radiology. The reference lists of the retrieved articles were checked for other relevant publications.

Data collection and analysis. Independent extraction of the data was performed by two authors. The extracted information included name of first author, publication date, number of patients, patient characteristics, study design/risk of bias, TNM stage and outcome. The data were entered into a standardized Excel file. Disagreements were resolved through discussion and consensus.

Time-to-event data from each study were summarized using the log hazard ratio (HR) and variance. When this information was not reported by the trials, it was estimated from data such as the log-rank test P-value (8), whereas timeto-event data were calculated from the Kaplan-Meier survival curves. Kaplan-Meier curves were interpreted with the Engauge Digitizer software, version 4.1. Data were combined using RevMan software, version 5.3. Pooling of the log HR and its variance was performed with the use of an inversevariance weighted mean, and the findings are presented as HR and $95 \%$ confidence interval $(95 \% \mathrm{CI})$.

The DerSimonian-Laird random effects analysis was used for estimating the differences in survival (9) by generating a collective survival difference with a $95 \%$ CI using a heterogeneity assay at each endpoint. The survival rate was calculated from the survival curves where it was not readily available in the text or tables. Subjects censored before each endpoint were subtracted from the denominators (patient no. during follow-up), providing a conservative CI for the summary statistic. Counting of the censored cases was performed via placement of tick marks on survival curves (10).

Heterogeneity across studies was evaluated with the use of the $\mathrm{I}^{2}$ statistic, which is considered as a quantitative measure of irregularity across studies. Studies with $\mathrm{I}^{2} 25-50 \%$ were considered as having low heterogeneity, whereas those with $\mathrm{I}^{2} 50-75 \%$ were considered as having reasonable heterogeneity and those with $\mathrm{I}^{2}>75 \%$ as having high heterogeneity (11).

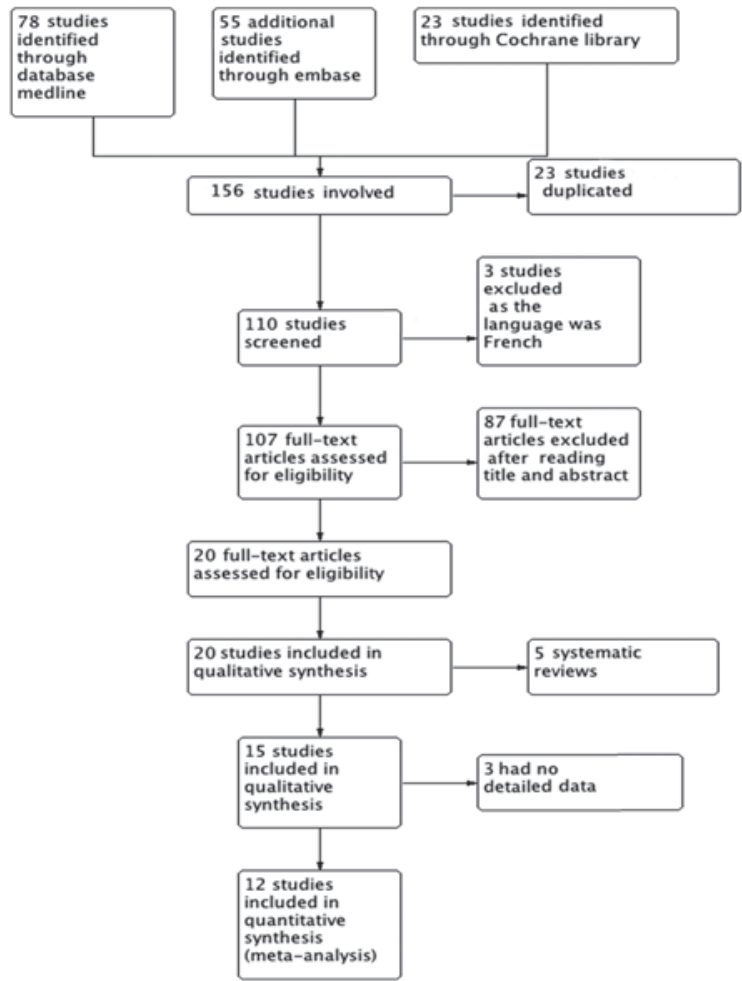

Figure 1. Flow diagram of the literature search and study selection process.

$\mathrm{I}^{2}>50 \%$ reflected significant heterogeneity (12). A fixed-effects model was applied unless there was significant unexplained heterogeneity, in which case a random-effects model was employed.

\section{Results}

A total of 156 citations were identified from the database search: 23 RCTs were excluded due to data duplication and 87 RCTs were excluded after reading the titles and abstracts. A full-text review of the remaining articles was performed, and 3 studies did not include relevant data in detail (13-15), 3 were published in French (16-19), and 5 more were excluded as they were systematic reviews (20-24). Finally, 12 RCTs (25-36) fulfilled our inclusion criteria and were entered in the present meta-analysis. The flowchart of the study inclusion process is shown in Fig. 1.

Of the 12 RCTs, 7 were on LC and 5 on hypopharyngeal cancer. All the RCTs compared patients receiving IC followed by locoregional therapy (laryngectomy and/or radiotherapy or concomitant radiotherapy and chemotherapy), vs. those undergoing locoregional therapy alone (laryngectomy and/ or radiotherapy or chemoradiotherapy). Despite the differences among these trials, such as duration of the study and/ or follow-up, the effect of study heterogeneity on the OS for LC as well as for hypopharyngeal cancer was not statistically significant $\left(\mathrm{I}^{2}=21 \%, \mathrm{P}=0.28\right.$; s 2$)$; moreover, no significant difference in OS was observed between patients who received and those who did not receive IC for hypopharyngeal cancer or laryngeal cancer $(\mathrm{HR}=1.04,95 \% \mathrm{CI}$ : 0.98-1.10, $\mathrm{P}=0.21$; Fig. 2). However, IC had better treatment outcomes for patients with LC compared with surgery $(\mathrm{HR}=1.30,95 \% \mathrm{CI}: 1.16-1.46$, 


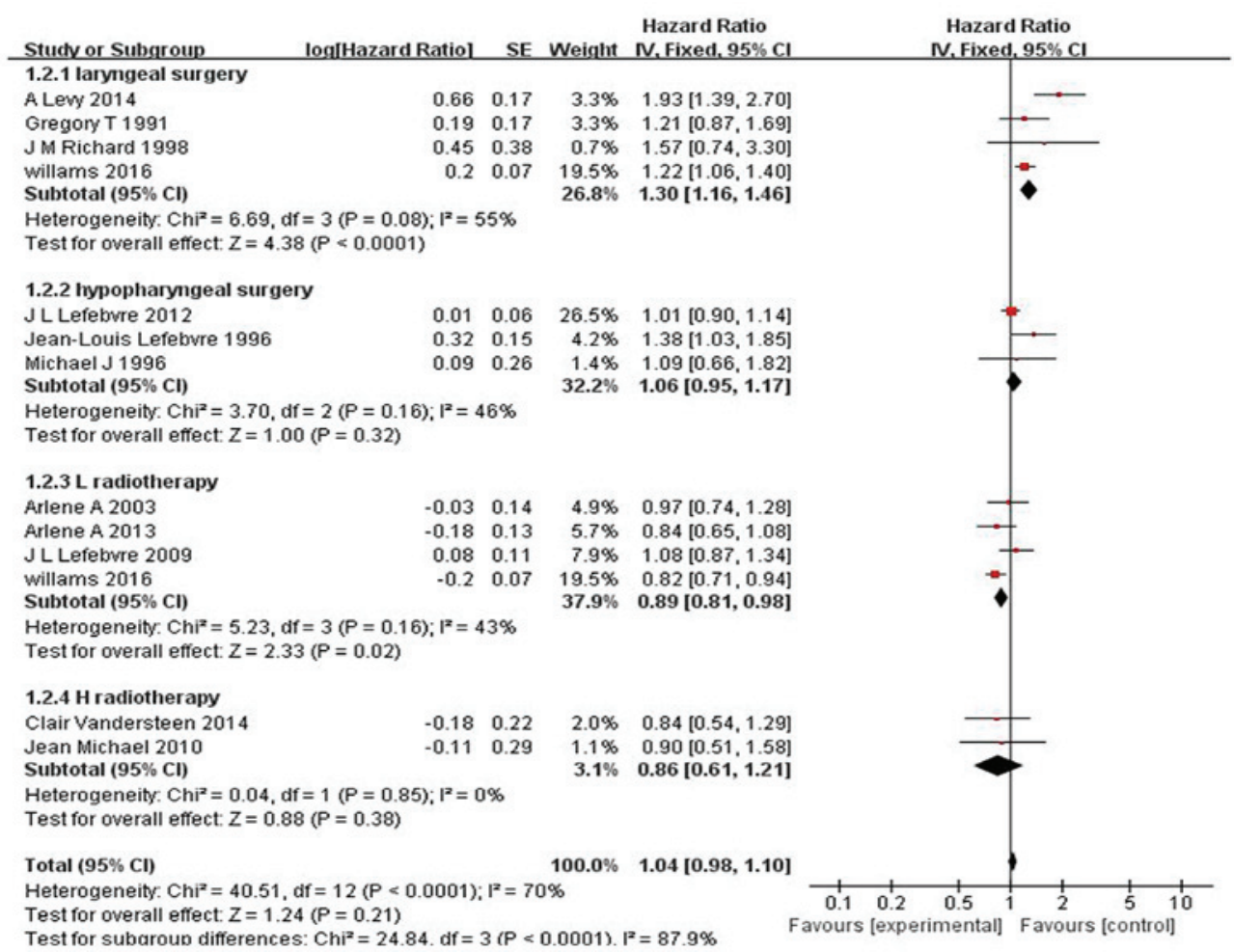

Figure 2. Hypopharyngeal cancer and laryngeal cancer overall survival. Metaanalysis of the comparison between laryngeal cancer and hypopharyngeal cancer patients receiving induction chemotherapy (experimental) and surgery or radiotherapy (control) in terms of overall survival. $\mathrm{R}$, radiotherapy; $\mathrm{R}+\mathrm{C}$, radiotherapy and chemotherapy or concurrent radiochemotherapy; CI, confidence interval.

$\mathrm{P}<0.0001$; Fig. 2), and IC may have result in more adverse side effects in laryngeal cancer compared with radiotherapy (HR=0.89, 95\% CI: 0.81-0.98, P=0.02; Fig. 2), However, as the number of cases in the present study was limited, these results require confirmation through further studies and large multicenter clinical trials.

There was obvious heterogeneity regarding disease-free survival (DFS) $\left(\mathrm{I}^{2}=88 \%, \mathrm{P}<0.00001\right)$ and laryngeal preservation (LP) $\left(\mathrm{I}^{2}=93 \%, \mathrm{P}<0.00001\right)$. Accordingly, the random-effects model was applied. The results for DFS were $\mathrm{HR}=1.24,95 \%$ CI: $0.99-1.56$ and $\mathrm{P}=0.06$ (Fig. 3) and for $\mathrm{LP} \mathrm{HR}=1.01$, 95\% CI: 0.72-1.40 and $\mathrm{P}=0.97$ (Fig. 4). Thus, IC exhibited higher efficacy vs. surgery in LC and vs. radiotherapy in hypopharyngeal cancer.

In the 12 RCTs on LC and hypopharyngeal cancer (including 4,320 patients) that focused on locoregional control, laryngeal preservation was not possible $(\mathrm{HR}=0.82,95 \% \mathrm{CI}: 0.76-0.88$, $\mathrm{P}<0.00001)$ following IC in responders, without a decrease in OS (Fig. 5).

No substantial difference was observed in the local recurrence rate between patients who did and those who did not receive $\mathrm{IC}(\mathrm{HR}=0.96,95 \% \mathrm{CI}: 0.90-1.02, \mathrm{P}=0.21)$ (Fig. 6). However, in hypopharyngeal carcinoma, the IC group exhibited a significantly lower long-term (5-year) rate of distant recurrence (difference of $11.7 \%$; $95 \% \mathrm{CI}: 10.3-13.3 \%, \mathrm{P}=0.02$ ) vs. surgery (Fig. 7).

\section{Discussion}

In the present study, it was demonstrated that IC was beneficial for patients with locally advanced and resectable LC, with a
$11.7 \%$ lower rate of distant metastasis. However, this conclusion may differ among different studies. Jie et al (3) also reported that the IC group had a lower rate of distant metastasis by $8 \%$ (95\% CI: 1-16, P=0.02). Furthermore, a randomized phase 3 trial (37) divided patients into two groups, one receiving IC followed by concurrent chemoradiotherapy $(n=70)$, and the other receiving concurrent chemoradiotherapy alone $(n=75)$; finally, $5(7 \%)$ patients in the induction group and $8(11 \%)$ in the concurrent chemoradiotherapy group developed distant metastasis. Their findings demonstrated that adding IC may be superior to concurrent chemoradiotherapy alone in the treatment of locally advanced head and neck squamous cell carcinoma (HNSCC). However, Su et al (38) conducted a meta-analysis in 2008, and included 4 RCTs reporting that the difference in distant metastasis between the treatment group and the control group was not significant, while 1 study reported that the difference was statistically significant. Thus, more large-scale RCTs and/or extensive meta-analyses are required. However, IC was not found to be associated with any major differences regarding local recurrence. Furthermore, the TAX324 study (34) reported that there was no significant difference in local and distant recurrence between the IC and control groups.

The combination of cisplatin and 5-fluorouracil (PF) was applied as IC. The use of docetaxel has been shown to improve OS rate, but this may due to the patients exhibiting different responses to IC. Human papillomavirus (HPV) infection, smoking, drinking, epidermal growth factor receptor (EGFR) expression and sex may act as prognostic factors in HNSCC (39). Among patients with oropharyngeal carcinoma, $64 \%$ were HPV-16 positive, and the age range was 55-63 years, 


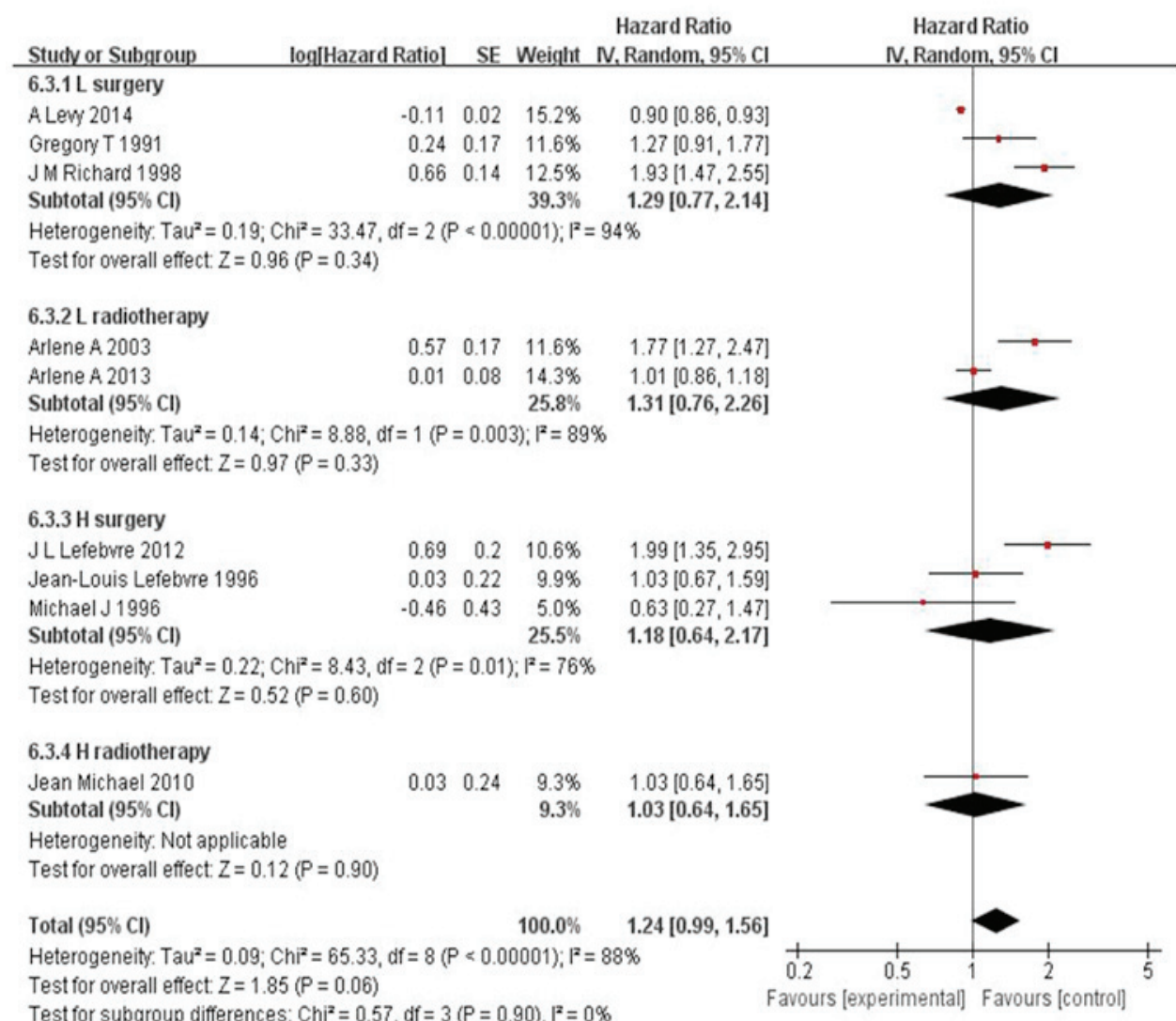

Figure 3. Disease-free survival. Metaanalysis of the comparison between patients receiving induction chemotherapy (experimental) and surgery or radiotherapy (control) in terms of disease-free survival. L, laryngeal; $\mathrm{H}$, hypopharyngeal; $\mathrm{R}+\mathrm{C}$, radiotherapy and chemotherapy or concurrent radiochemotherapy; CI, confidence interval.

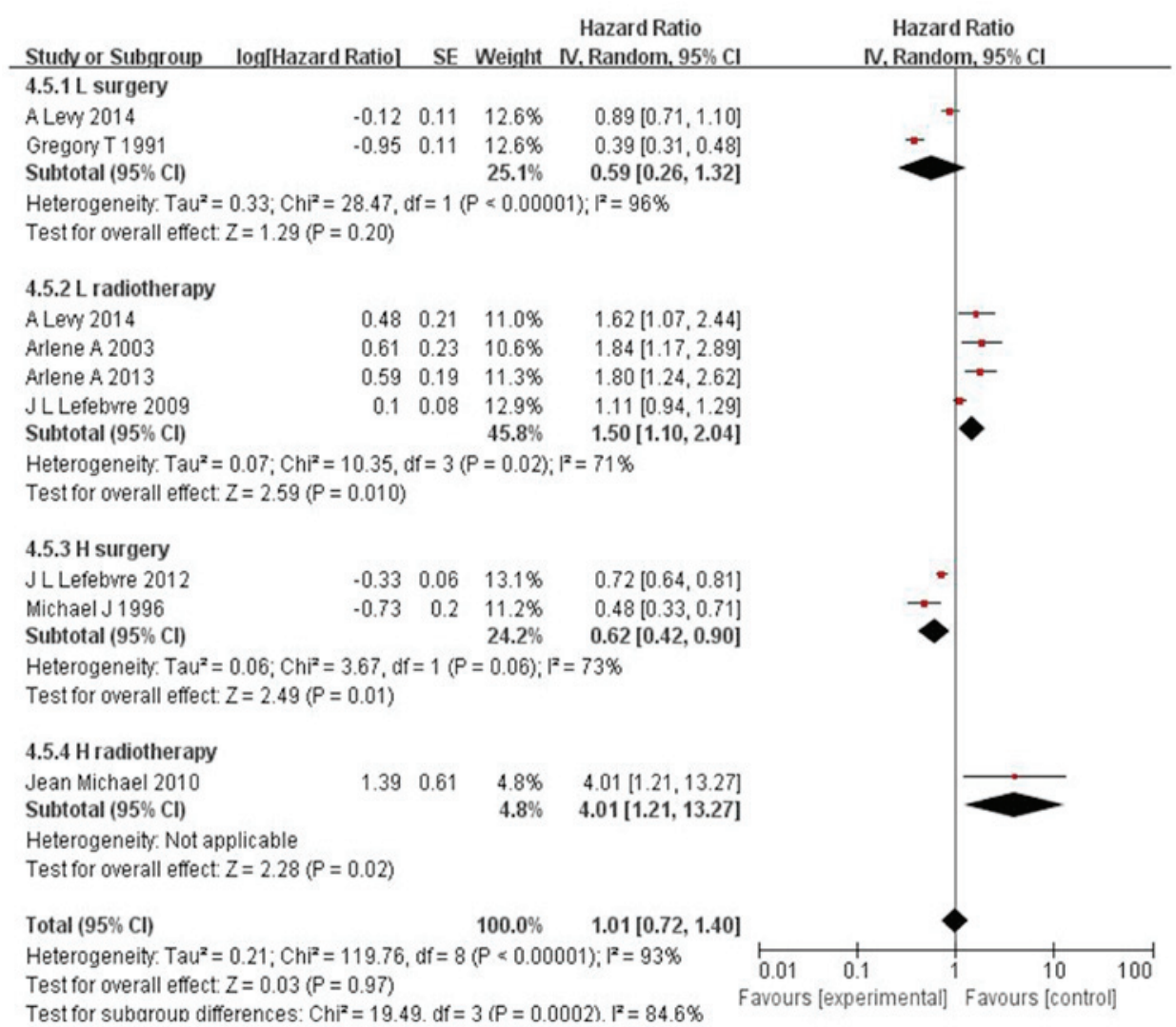

Figure 4. Laryngeal preservation. Metaanalysis of the comparison between patients with induction chemotherapy (experimental) and surgery or radiotherapy (control) in terms of laryngeal preservation. CI, confidence interval. 


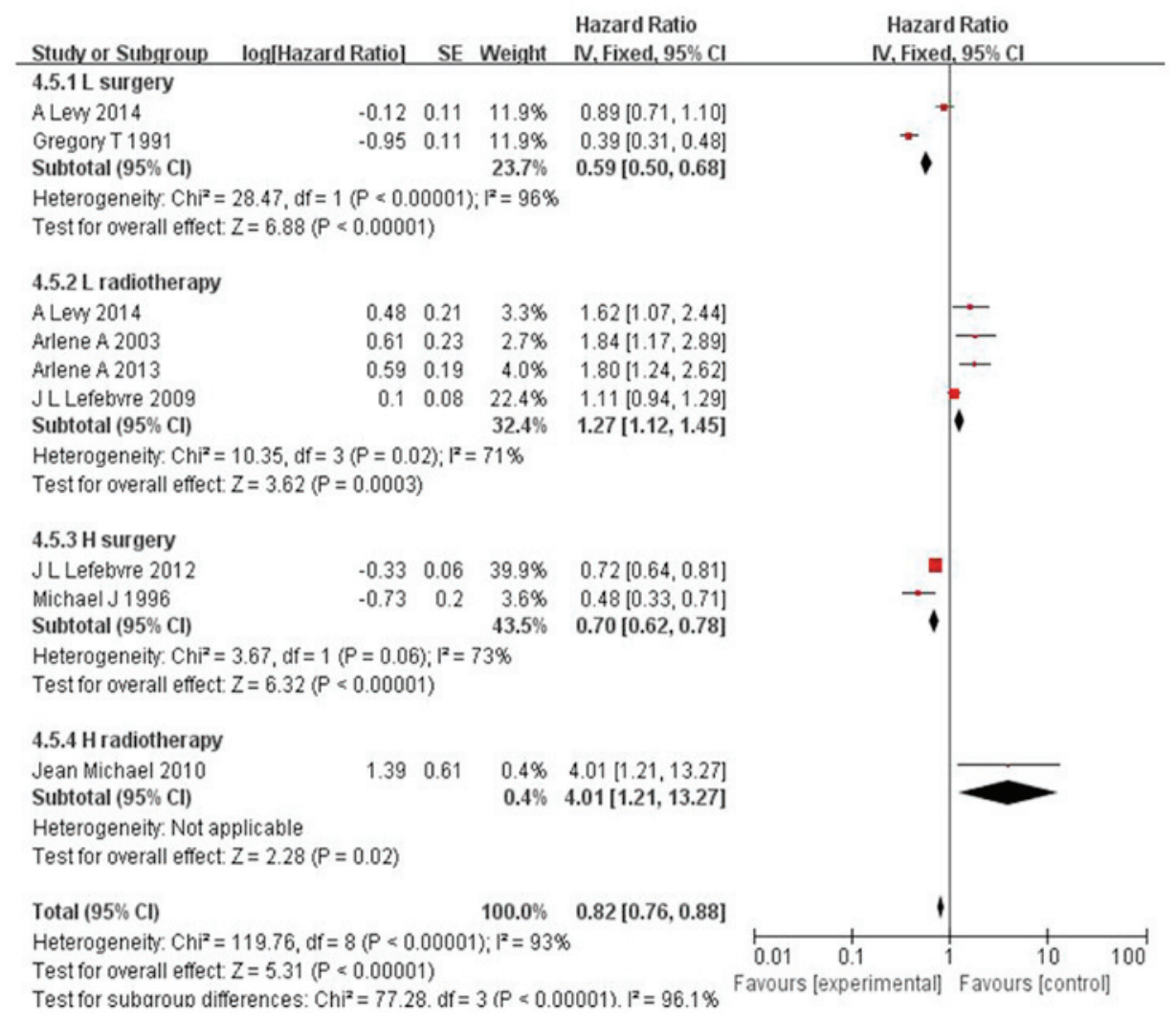

Figure 5. Locoregional control. Metaanalysis of the comparison between patients receiving induction chemotherapy (experimental) and surgery or radiotherapy (control) in terms of locoregional control. L, laryngeal; H, hypopharyngeal; CI, confidence interval.

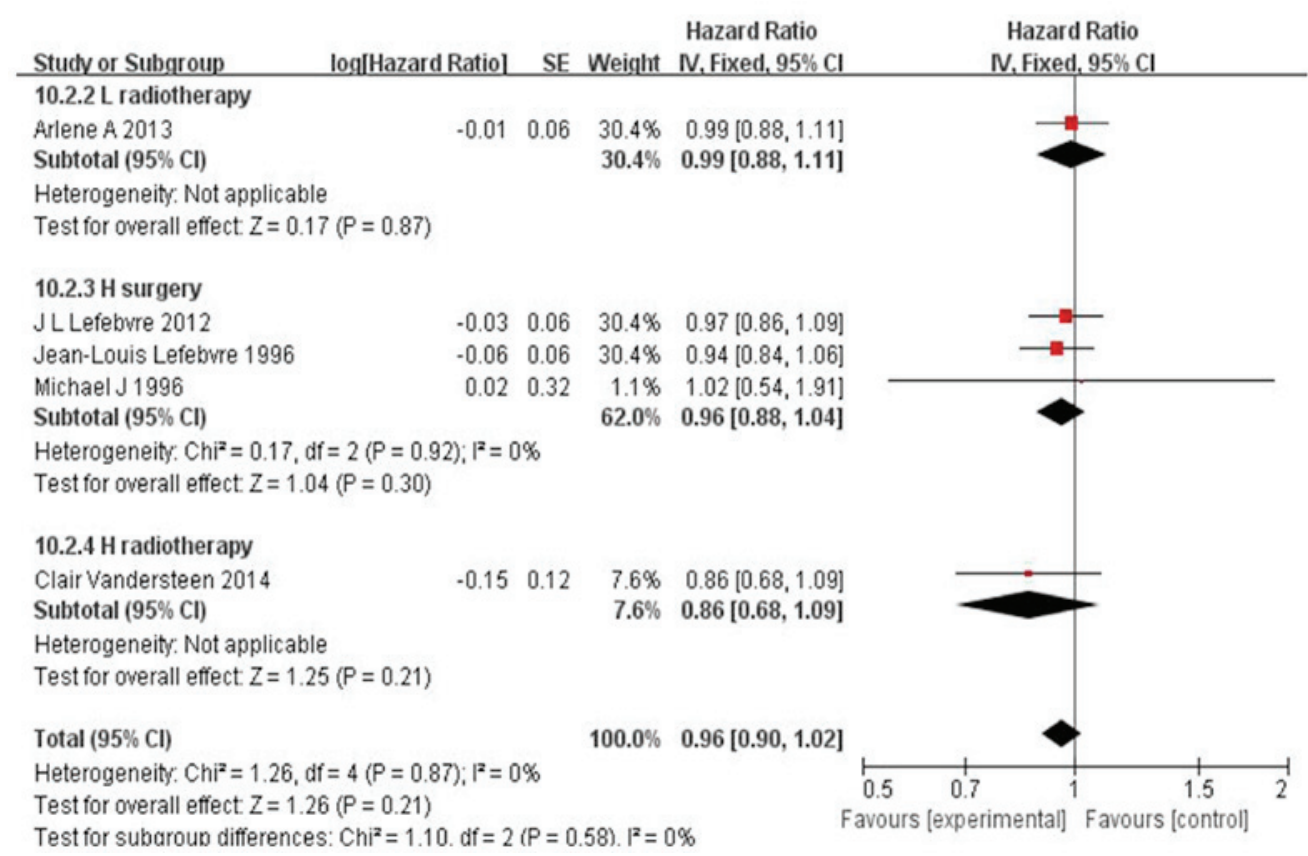

Figure 6. Local recurrence. Metaanalysis of the comparison between patients with induction chemotherapy (experimental) and surgery or radiotherapy (control) regarding local recurrence. L, laryngeal; H, hypopharyngeal; CI, confidence interval.

with positive subjects being younger compared with negative subjects. Men are more susceptible compared with women (73.3 vs. $41.6 \%$, respectively). The degree of HPV infection and virus subtype was obviously associated with the response to IC and better OS and disease-specific survival. High EGFR expression was also associated with poor response to IC and poor OS. In the present study, smoking appeared to be significantly associated with higher EGFR expression and lower HPV load. The abovementioned factors may affect the OS rate of patients with $\mathrm{LC}$ and the extent of response to IC. The additive effect of lower 


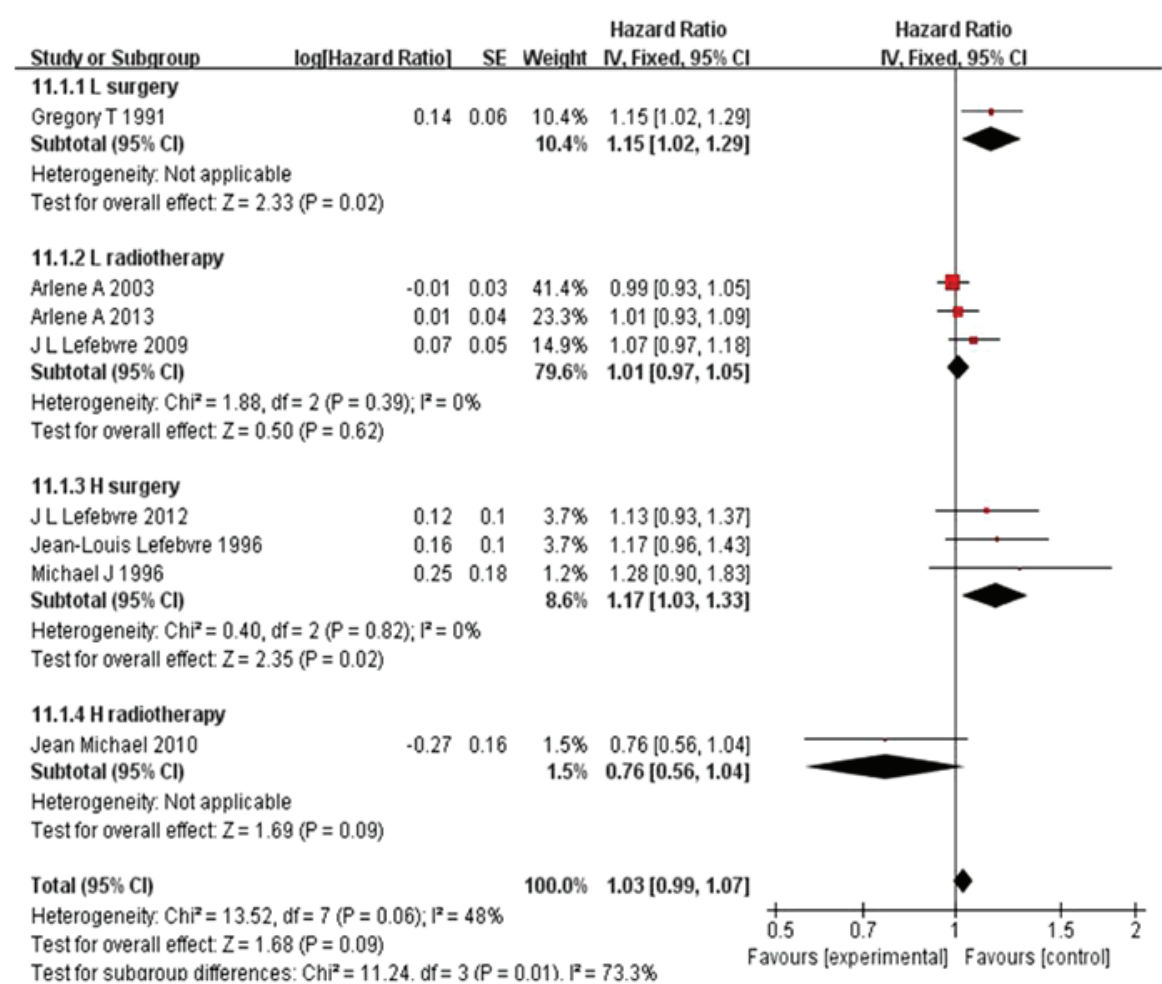

Figure 7. Distant recurrence. Metaanalysis of the comparison between patients with induction chemotherapy (experimental) and surgery or radiotherapy (control) regarding their distant recurrence, $\mathrm{L}$, laryngeal; $\mathrm{H}$, hypopharyngeal; $\mathrm{CI}$, confidence interval.

EGFR expression and higher HPV titer was associated with better OS and disease-specific survival. HPV-negative tumors or those with higher EGFR expression had the worst OS and disease-specific survival, as all patients (10/10) succumbed to the disease within 2.5 years. However, IC appears to be beneficial in terms of DFS. The impact of IC is likely to differ according to the location of the tumor. Currently, in patients with resectable locally advanced hypopharyngeal cancer, surgery, radiotherapy or chemoradiotherapy are considered as the standard treatments. As shown in Fig. 2, in patients with hypopharyngeal cancer, IC is likely to favorably affect OS rate compared with surgery. However, the result does not appear to be consistent with the conventional belief in respect of the sequential or concurrent chemoradiotherapy, as it appears that IC is more effective in LC compared with hypopharyngeal cancer. As regards LC, IC may be beneficial in terms of OS rate in patients with resectable disease, which has also been suggested by other meta-analyses $(4,6,7)$. This may be due to a number of factors, such as the heterogeneity of the patients and the location of the tumor, and the data of the present study may not suffice. Therefore, it is necessary to analyze the factors associated with the response to IC in patients with advanced LC.

In addition, $\mathrm{PF}+$ docetaxel (TPF) is hypothesized to be the optimal IC choice for the control of LC patients, which is likely to be due to the fact that squamous cell carcinoma is sensitive to docetaxel and $95 \%$ of LC cases are squamous cell carcinomas. However, it has not been elucidated whether induction TPF enhances resectability on provision of priority to surgery in patients with locally advanced and resectable LC, which has been reported previously. The TAX324 study suggested that IC with TPF confers a long-term survival benefit compared with PF in locally advanced head and neck cancer. Therefore, it is recommended that patients who are candidates for IC ARE treated with TPF. In 2010, Calais (40) added the taxane docetaxel to PF, creating the TPF triplet regimen, which achieved significantly higher laryngeal preservation and laryngectomy-free survival rates compared with the PF doublet regimen. TPF is currently the accepted standard IC regimen in clinical trials including patients with resectable disease. However, Levy et al (32) reported that the addition of taxanes did not improve outcome in their series. Levy et al questioned the validity of the results of certain studies, such as the TAX324 trial (34), as it only included a total of 35 patients; thus, the interpretation of these results should be performed with caution and more studies are required to clearly determine the role of taxanes in this setting.

A number of laboratory trials validated the benefits associated with EGFR-targeted agents for the treatment of locally advanced and resectable LC. Traditional Chinese medicines, such as curcumin and resveratrol, are also currently applied in the treatment of head and neck cancer (41-43), in addition to being reported to be effective in tumors in other locations (44). Predictive biomarkers that reflect the efficacy and safety of IC are expected to assist with treatment selection, or used to determine whether IC must be performed, particularly in resectable lesions. In cases where the biomarkers predict disadvantage to IC, it should not be performed; otherwise, IC may be beneficial in terms of survival rate. Potential biomarkers may include DNA gene mutations, epigenetic variations, as well as levels of mRNA or protein expression (45). It was discovered that the mechanism underlying the antitumor effects of liriodenine is likely mediated via upregulation of p53 expression, which eventually stimulates cell apoptosis (45). p53 gene changes are strongly associated with low risk outcomes in PF-based 
IC, which suggests that patients with LC must first undergo screening for p53 changes prior to the selection of the most suitable treatment protocol. An international team (46) conducted a genome-wide association study (GWAS) on 993 patients with squamous cell LC and 1,995 cancer-free controls from Chinese communities, and identified three novel susceptibility loci at 11q12 (rs174549), 6p21 (rs2857595) and 12q24 (rs10492336). This was the first global cancer research applying GWAS, the results of which are expected to further advance the research on the mechanism of LC, aiding early identification, timely diagnosis and molecular targeted therapy of LC. In addition, Liang et al (47) conducted a study on dendritic cell (DC) fusion vaccine that acts on human laryngeal carcinoma HEp-2 cells. In the present study, it was revealed that that SOCS1 siRNA and IL-12 gene modified DCs together, which may provide novel strategies for polygenic therapy in LC. To the best of our knowledge, this is the first study on this subject available in the literature to date. Therefore, additional investigation is required to elucidate whether IC can enable organ preservation in non-laryngeal locations.

The number of related studies on LC is limited, and the sample size of the present study is small; therefore, the results must be interpreted with caution. In the present study, 3 articles reported patient withdrawals. Due to non-standardized cases with loss to follow-up and incomplete records, the actual and long-term curative efficacy of IC should be interpreted with caution, pending further research. The sample size of each RCT included in this study was satisfactory, and all RCTs reported adverse reactions in terms of mucosal damage caused by IC, with salivary gland, pharyngeal, esophageal and laryngeal toxicity being the most common serious events. These complications were associated with fatalities in all groups. In addition, attention must be paid to the possibility of IC-related kidney injury with ensuing renal functional changes. In the late-stage clinical studies, attention should be paid to the following: Applying the randomization principle, ensuring balance between groups, thereby improving the credibility of the research results; using blinding methods to reduce information bias; correct estimation of the sample size to reduce the sampling error; and censoring of withdrawals and cases lost to follow-up. In addition, the number of cases and the reasons for analysis should be provided; rigorous scientific inclusion and exclusion criteria should be established to ensure high quality of the RCTs; attention must be paid to the monitoring and recording of adverse reactions to better evaluate the safety of the interventions. In conclusion, IC confers an advantage in terms of lowering the rate of distant metastases, in addition to prolonging DFS, enabling laryngeal preservation and increasing the OS rate in the patients with locally advanced and resectable LC. However, there is no sufficient evidence to support its superiority in terms of locoregional control and local recurrence. More studies on laryngeal preservation are required to optimize IC protocols; moreover, additional molecular biomarkers are required to identify patients that are likely to respond to $\mathrm{IC}$.

\section{Acknowledgements}

Not applicable.

\section{Funding}

No funding was received.

\section{Availability of data and materials}

The analyzed data sets generated during the present study are available from the corresponding author on reasonable request.

\section{Ethics approval and consent to participate}

Not applicable.

\section{Consent for publication}

All patients provided consent for publication.

\section{Authors' information}

XFW made substantial contributions to the conception and design of the study, as well as the acquisition, analysis and interpretation of data. LG gave final approval of the version to be published and made substantial contributions to the conception and design of the study. PG contributed in drafting the manuscript and revising it critically for important intellectual content, as well as the collection of data.

\section{Competing interests}

The authors declare that they have no competing interests.

\section{References}

1. Forastiere AA: Head and neck cancer: Overview of recent developments and future directions. Semin Oncol 27 (Suppl 8): 1-4, 2000.

2. Posner MR, Hershock DM, Blajman CR, Mickiewicz E, Winquist E, Gorbounova V, Tjulandin S, Shin DM, Cullen K, Ervin TJ, et al; TAX 324 Study Group: Cisplatin and fluorouracil alone or with docetaxel in head and neck cancer. N Engl J Med 357: 1705-1715, 2007.

3. Jie M, Ying L, Xi Y, et al: Induction chemotherapy inpatients with resectable head and neck squamous cell carcinoma: A Meta-analysis. World J Surg Oncol 11: 61-67, 2013.

4. Pignon JP, Bourhis J, Domenge C and Designé L: Chemotherapy added to locoregional treatment for head and neck squamouscell carcinoma: Three meta-analyses of updated individual data. MACH-NC Collaborative Group. Meta-Analysis of Chemotherapy on Head and Neck Cancer. Lancet 355: 949-955, 2000.

5. Monnerat C, Faivre S, Temam S, Bourhis J and Raymond E: End points for new agents in induction chemotherapy for locally advanced head and neck cancers. Ann Oncol 13: 995-1006, 2002.

6. Pignon JP, le Maître A, Maillard E and Bourhis J; MACH-NC Collaborative Group: Meta-analysis of chemotherapy in head and neck cancer (MACH-NC): An update on 93 randomised trials and 17,346 patients. Radiother Oncol 92: 4-14, 2009.

7. Glenny AM, Furness S, Worthington HV, Conway DI, Oliver R, Clarkson JE, Macluskey M, Pavitt S, Chan KK and Brocklehurst P; CSROC Expert Panel: Interventions for the treatment of oral cavity and oropharyngeal cancer: Radiotherapy. Cochrane Database Syst Rev 4: CD006387, 2010.

8. Parmar MKB, Torri V and Stewart L: Extracting summary statistics to perform meta-analyses of the published literature for survival endpoints. Stat Med 17: 2815-2834, 1998.

9. DerSimonian R and Laird N: Meta-analysis in clinical trials. Control Clin Trials 7: 177-188, 1986.

10. Mitsudomi T, Hamajima N, Ogawa M and Takahashi T: Prognostic significance of p53 alterations in patients with non-small cell lung cancer: a meta-analysis. Clin Cancer Res 6: 4055-4063, 2000. 
11. Higgins JP, Thompson SG, Deeks JJ and Altman DG: Measuring inconsistency in meta-analyses. BMJ 327: 557-560, 2003.

12. Armitage P, Berry G, Matthews JNS: Analysing means and proportions. In: Statistical Methods in Medical Research. 4th edition. Blackwell Science Ltd., Hoboken, NJ, pp83-146, 2008

13. Olsen KD: Reexamining the treatment of advanced laryngeal cancer. Head Neck 32: 1-7, 2010.

14. Jarząb A, Grabarska A, Kiełbus M, Jeleniewicz W, DmoszyńskaGraniczka M, Skalicka-Woźniak K, Sieniawska E, Polberg K and Stepulak A: Osthole induces apoptosis, suppresses cell-cycle progression and proliferation of cancer cells. Anticancer Res 34 6473-6480, 2014.

15. Dewyer NA, Wolf GT, Light E, Worden F, Urba S, Eisbruch A, Bradford CR, Chepeha DB, Prince ME, Moyer J, et al: Circulating CD4-positive lymphocyte levels as predictor of response to induction chemotherapy in patients with advanced laryngeal cancer. Head Neck 36: 9-14, 2014

16. Bourhis J, Lefebvre JL, Temam S, Lusinchi A, Janot F, Wibault P, and Pignon JP: Laryngeal preservation: nonsurgical approaches. Cancer Radiother 8: S24-S28, 2004 (In French).

17. Pointreau Y,Lafond C, LegoutéF, Trémolières $P$, Servagi-Vernat $S$, Giraud P, Maingon P, Calais G and Lapeyre M: Radiotherapy of larynx cancers. Cancer Radiother 20 (Suppl): S131-S135, 2016 (In French).

18. Calais G, Chapet S, Ruffier-Loubière A and Bernadou G: Induction chemotherapy for locally advanced head and neck cancer. Cancer Radiother 17: 498-501, 2013 (In French).

19. Lévy A, Blanchard P, Janot F, Temam S, Bourhis J, DalySchveitzer N and Tao Y: Results of definitive radiotherapy for squamous cell carcinomas of the larynx patients with subglottic extension. Cancer Radiother 18: 1-6, 2014 (In French).

20. Forastiere AA, Weber RS and Trotti A: Organ Preservation for Advanced Larynx Cancer: Issues and Outcomes. J Clin Oncol 33: 3262-3268, 2015

21. Toohill RJ, Duncavage JA, Grossmam TW, Malin TC, Teplin RW, Wilson JF, Byhardt RW, Haas JS, Cox JD, Anderson T, et al: The effects of delay in standard treatment due to induction chemotherapy in two randomized prospective studies. Laryngoscope 97: 407-412, 1987

22. Carew JF and Shah JP: Advances in multimodality therapy for laryngeal cancer. CA Cancer J Clin 48: 211-228, 1998.

23. Gilbert $\mathrm{J}$ and Forastiere A: Organ preservation trials for laryngeal cancer. Otolaryng Clin N Am 35: 1035-1054, 2002.

24. Gorphe P, Matias M, Even C, Ferte C, Bidault F, Garcia G Temam S, Nguyen F, Blanchard P, Tao Y, et al: Laryngo-esophageal Dysfunction-free Survival in a Preservation Protocol for T3 Laryngeal Squamous-cell Carcinoma. Anticancer Res 36: 6625-6630, 2016.

25. Wolf GT, Fisher SG, Hong WK, Hillman R, Spaulding M, Laramore GE, Endicott JW, McClatchey K and Henderson WG; Department of Veterans Affairs Laryngeal Cancer Study Group: Induction chemotherapy plus radiation compared with surgery plus radiation in patients with advanced laryngeal cancer. $\mathrm{N}$ Engl J Med 324: 1685-1690, 1991.

26. Zelefsky MJ, Kraus DH, Pfister DG, Raben A, Shah JP, Strong EW, Spiro RH, Bosl GJ and Harrison LB: Combined chemotherapy and radiotherapy versus surgery and postoperative radiotherapy for advanced hypopharyngeal cancer. Head Neck 18: 405-411, 1996.

27. Richard JM, Sancho-Garnier H, Pessey JJ, Luboinski B Lefebvre JL, Dehesdin D, Stromboni-Luboinski M and Hill C: Randomized trial of induction chemotherapy in larynx carcinoma. Oral Oncol 34: 224-228, 1998.

28. Forastiere A, Goepfert H, Maor M, Pajak T, Webwe R, Morrison W, et al: Concurrent chemotherapy and radiotherapy for organ preservation in advanced laryngeal cancer. N Engl J Med 349: 2091-2098, 2003

29. Prades JM, Lallemant B, Garrel R, Reyt E, Righini C, Schmitt T, Remini N, Saban-Roche L, Timoshenko AP, Trombert B, et al: Randomized phase III trial comparing induction chemotherapy followed by radiotherapy to concomitant chemoradiotherapy for laryngeal preservation in T3M0 pyriform sinus carcinoma. Acta Otolaryngol 130: 150-155, 2010.

30. Lefebvre JL, Andry G, Chevalier D, Luboinski B, Collette L, Traissac L, de Raucourt D, Langendijk JA; EORTC Head and Neck Cancer Group: Laryngeal preservation with induction chemotherapy for hypopharyngeal squamous cell carcinoma: 10-year results of EORTC trial 24891. Ann Oncol 23: 2708-2714, 2012.
31. Forastiere AA, Zhang Q, Weber RS, Maor MH, Goepfert H, Pajak TF, Morrison W, Glisson B, Trotti A, Ridge JA, Thorstad W, et al: Long-term results of RTOG 91-11: a comparison of three nonsurgical treatment strategies to preserve the larynx in patients with locally advanced larynx cancer. J Clin Oncol 31: 845, 2013

32. Levy A, Blanchard P, Temam S, Maison MM, Janot F, Mirghani H, Bidault F, Guigay J, Lusinchi A, Bourhis J, et al: Squamous cell carcinoma of the larynx with subglottic extension: Is larynx preservation possible? Strahlenther Onkol 190: 654-660, 2014.

33. Vandersteen C, Benezery K, Chamorey E, Ettaiche M, Dassonville $O$, Poissonnet $G$, Riss JC, Pierre CS, Hannoun-Lévi JM, Chand ME, et al: Contemporary therapeutic management of locally advanced hypopharyngeal cancer: Oncologic and functional outcomes - a report on 100 cases. Acta Otolaryngol 135: 193-200, 2015.

34. Lorch JH, Goloubeva O, Haddad RI, Cullen K, Sarlis N, Tishler R, Tan M, Fasciano J, Sammartino DE and Posner MR TAX 324 Study Group: Induction chemotherapy with cisplatin and fluorouracil alone or in combination with docetaxel in locally advanced squamous-cell cancer of the head and neck: Long-term results of the TAX 324 randomised phase 3 trial. Lancet Oncol 12: 153-159, 2011.

35. Lefebvre JL, Chevalier D, Luboinski B, Kirkpatrick A, Collette L and Sahmoud T: Larynx preservation in pyriform sinus cancer: preliminary results of a European Organization for Research and Treatment of Cancer phase III trial. EORTC Head and Neck Cancer Cooperative Group. Journal of the National Cancer Institute 8: 890-899, 1996.

36. Lefebvre JL, Rolland F, Tesselaar M, Bardet E, Leemans CR, Geoffrois L, Hupperets P, Barzan L, de Raucourt D, Chevalier D, et al; EORTC Head and Neck Cancer Cooperative Group; EORTC Radiation Oncology Group: Phase 3 randomized trial on larynx preservation comparing sequential vs alternating chemotherapy and radiotherapy. J Natl Cancer Inst 101: 142-152, 2009.

37. Haddad R, O'Neill A, Rabinowits G, Tishler R, Khuri F, Adkins D, Clark J, Sarlis N, Lorch J, Beitler JJ, et al: Induction chemotherapy followed by concurrent chemoradiotherapy (sequential chemoradiotherapy) versus concurrent chemoradiotherapy alone in locally advanced head and neck cancer (PARADIGM): A randomised phase 3 trial. Lancet Oncol 14: 257-264, 2013.

38. Su YX, Zheng JW, Zheng GS, Liao GQ and Zhang ZY: Neoadjuvant chemotherapy of cisplatin and fluorouracil regimen in head and neck squamous cell carcinoma: A meta-analysis. Chin Med J (Engl) 121: 1939-1944, 2008

39. Kumar B, Cordell KG, Lee JS, et al: Response to Therapy and Outcome in Oropharyngeal Cancer are Associated with Biomarkers Including HPV, EGFR, Gender and Smoking. Int J Radiat Oncol Biol Phys 69 (Suppl 1): 109, 2007.

40. Calais G: TPF: a rational choice for larynx preservation? Oncologist, 2010, 15 Suppl 3 (15 Suppl 3):19-24.

41. Wilken R, Veena MS, Wang MB and Srivatsan ES: Curcumin: A review of anti-cancer properties and therapeutic activity in head and neck squamous cell carcinoma. Mol Cancer 10: 12, 2011.

42. Baumeister P, Reiter M and Harréus U: Curcumin and Other Polyphenolic Compounds in Head and Neck Cancer Chemoprevention. Oxid Med Cell Longev2012:902716, 2012.

43. Qiao Y, Gao K, Wang Y, Wang X and Cui B: Resveratrol ameliorates diabetic nephropathy in rats through negative regulation of the p38 MAPK/TGF- $\beta 1$ pathway. Exp Ther Med 13: 3223-3230, 2017

44. Ghalandarlaki N, Alizadeh AM and Ashkani-Esfahani S: Nanotechnology-applied curcumin for different diseases therapy. Biomed Res Int 2014: 394264, 2014.

45. Li L, Xu Y and Wang B: Liriodenine induces the apoptosis of human laryngocarcinoma cells via the upregulation of p53 expression. Oncol Lett 9: 1121-1127, 2015.

46. Wei Q, Yu D, Liu M, Wang M, Zhao M, Liu M, Jia W, Ma H, Fang J, Xu W, et al: Genome-wide association study identifies three susceptibility loci for laryngeal squamous cell carcinoma in the Chinese population. Nat Genet 46: 1110-1114, 2014.

47. Liang $W$ and Wang XF: In vitro induction of specific anti-tumoral immunity against laryngeal carcinoma by using human interleukin-12 gene-transfected dendritic cells. Chin Med J (Engl) 124: 1357-1361, 2011. 